\title{
Distúrbios da coagulação em pacientes obstétricas infectadas pelo SARS-CoV-2 (COVID-19)
}

\author{
Coagulation disorders in obstetric patients infected with SARS-CoV-2 (COVID-19) \\ Trastornos de la coagulación en pacientes obstétricas infectadas con SARS-CoV-2 \\ (COVID-19)
}

Laila Naiane da Silva Bahia ${ }^{1 *}$, Lavínia Prado Artiaga ${ }^{1}$, Mariana Souza Almeida ${ }^{1}$, Thaynara Rodrigues de Paula ${ }^{1}$, Pablinny Moreira Galdino de Carvalho' ${ }^{1}$.

\section{RESUMO}

Objetivo: Analisar os distúrbios coagulativos que ocorrem em grávidas infectadas pelo SARS-CoV-2, com o intuito de verificar quais são os fatores que predispõem as gestantes ao agravamento da COVID-19. Revisão bibliográfica: Ao analisar se a COVID-19 pode influenciar nos processos coagulativos que ocorrem fisiologicamente durante a gestação, constatou-se que a "tempestade de citocinas", ou seja, o estado hiperinflamatório causado pelo vírus, contribui para o agravamento da situação hipercoagulativa da gestação. Evidenciou-se, também, que há uma elevação de D-dímero acima do esperado para a gestação, com formação de microtrombos placentários, indicando um pior prognóstico materno e fetal. Verificou-se que há maiores desfechos negativos envolvidos, porém não está esclarecido que o agravo ocorra, necessariamente, por conta da infecção pelo SARS-CoV-2. Considerações finais: Ainda há uma carência de estudos sobre os agravos da COVID-19 em gestantes, porém, é importante ressaltar a necessidade de uma atenção maior para essas pacientes, em decorrência das alterações gestacionais que podem agravar o quadro da doença.

Palavras-chave: COVID-19, Coagulação, Gestantes.

\begin{abstract}
Objective: To analyze the coagulative disorders that occur in pregnant women infected with SARS-CoV-2, in order to verify which are the factors that predispose pregnant women to the worsening of COVID-19. Bibliographic review: When analyzing whether COVID-19 can influence the coagulative processes that occur physiologically during pregnancy, it was found that the "cytokine storm", that is, the hyperinflammatory state caused by the virus, contributes to the worsening of the hypercoagulative situation of pregnancy. It was also evident that there is an elevation of D-dimer above the expected for pregnancy, with the formation of placental microthrombi, indicating a worse maternal and fetal prognosis. It was found that there are greater negative outcomes involved, however it is not clear that the disease occurs, necessarily, due to the SARS-CoV-2 infection. Final considerations: There is still a lack of studies on the problems of COVID-19 in pregnant women, however, it is important to emphasize the need for greater attention for these patients, due to the gestational changes that can aggravate the disease.
\end{abstract}

Key words: COVID-19, Coagulation, Pregnant women.

\section{RESUMEN}

Objetivo: Analizar los trastornos de la coagulación que se presentan en gestantes infectadas con SARS-CoV2 , con el fin de verificar cuáles son los factores que predisponen a las gestantes al agravamiento del COVID-

${ }^{1}$ Universidade Federal do Oeste da Bahia (UFOB), Barreiras - BA. *E-mail: naianelaila@gmail.com SUBMETIDO EM: 3/2021

PUBLICADO EM: 5/2021 
19. Revisión bibliográfica: Al analizar si el COVID-19 puede influir en los procesos coagulativos que ocurren fisiológicamente durante el embarazo, se encontró que la "tormenta de citoquinas", es decir, el estado hiperinflamatorio provocado por el virus, contribuye al agravamiento de la situación hipercoagulativa de el embarazo. También se evidenció que existe una elevación del dímero $D$ por encima de lo esperado para el embarazo, con formación de microtrombos placentarios, lo que indica un peor pronóstico materno y fetal. Se encontró que hay mayores resultados negativos involucrados, sin embargo, no está claro que la enfermedad se produzca, necesariamente, debido a la infección por SARS-CoV-2. Consideraciones finales: Aún faltan estudios sobre los problemas del COVID-19 en mujeres embarazadas, sin embargo, es importante enfatizar la necesidad de una mayor atención para estas pacientes, debido a los cambios gestacionales que pueden agravar la enfermedad.

Palabras clave: COVID-19, Coagulación, Gestantes.

\section{INTRODUÇÃO}

A doença infecciosa ocasionada pelo novo coronavírus SARS-CoV-2 (COVID-19), recém descoberto, foi elevada à categoria de pandemia pela World Health Organization (WHO) em março do ano de 2020, sendo responsável por mais de 89 milhões de casos até janeiro de 2021. A principal forma de contágio é através de gotículas de saliva ou secreção nasal de uma pessoa infectada. Os sintomas mais comuns incluem: tosse seca, febre e cansaço. Sendo que os mais graves incluem dificuldade respiratória, dor pressórica no tórax, perda da fala ou movimento (WHO, 2020).

Assim como acontece na maioria das infecções, a COVID-19 ocasiona um aumento do estado inflamatório corporal, tendo como uma das principais respostas laboratoriais o aumento de D-dímero (marcador da geração de trombina e fibrinólise) gerando uma inflamação exacerbada, chamada de "tempestade de citocinas", diretamente proporcional à gravidade do quadro. Uma das consequências desse processo, é a alteração na coagulação que desempenha um papel patogênico por ocasionar a formação de trombos em vários órgãos, diminuindo a irrigação sanguínea e agravando a lesão local (BENHAMOU D, et al., 2020).

As mulheres grávidas também são suscetíveis ao SARS-COV-2, estando entre os grupos vulneráveis à doença. Elas ainda possuem alta suscetibilidade para o desenvolvimento de hipertensão e diabetes, ambos fatores de risco para os agravos causados pelo novo coronavírus (MAKATSARIYA AD, et al., 2020). De acordo com o Centro de Controle de Prevenção de Doenças dos Estados Unidos, as grávidas com COVID19 não apresentam risco aumentado de morte, no entanto, são mais propensas a serem hospitalizadas e necessitar de Unidade de Terapia Intensiva (UTI) e ventilação mecânica em relação às não grávidas (D'SOUZA R, et al., 2020).

A gravidez é um período em que muitas mudanças fisiológicas ocorrem em um curto espaço de tempo, sendo o sistema cardiovascular um dos mais afetados. Dentre essas alterações, é gerado um estado de hipercoagulação, diminuição do fluxo venoso, com aumento da capacitância venosa, compressão das veias pélvicas pelo aumento do útero gravídico e danos endoteliais devido às distensões venosas. Todas essas mudanças são responsáveis por proteger a mulher de hemorragias durante o parto. Entretanto, mesmo fisiológicas, essas alterações aumentam de 5 a 6 vezes o risco de tromboembolismo venoso, sendo necessário o perfeito funcionamento dos sistemas compensatórios do corpo, como o sistema anticoagulativo, fibrinolíticos e os fatores vasculares - óxido nítrico, prostaciclinas, dentre outros (MAKATSARIYA AD, et al., 2020).

Neste contexto, pode-se perceber que a infecção por COVID-19 durante a gravidez está associada a um alto risco de complicações trombóticas, devido ao estado de hipercoagulabilidade gravídico intrínseco e ao estado hiperinflamatório que altera a cascata coagulativa durante a infecção pelo SARS-CoV-2. Dessa maneira, pacientes gestantes graves infectadas pelo novo coronavírus podem desenvolver coagulopatias com bloqueio da microcirculação, ocasionando bloqueio de perfusão e podendo levar à falência múltipla de órgãos. (MAKATSARIYA AD, et al., 2020). 
Portanto, essa revisão teve como objetivo analisar a ocorrência de coagulopatias e modificações hematológicas devido à COVID-19 em pacientes obstétricas a fim de reunir informações importantes sobre os principais estudos, além de estabelecer a relação entre as alterações coagulativas inerentes à gestação com as complicações hemostáticas ocasionadas pelo SARS-CoV-2.

\section{REVISÃO BIBLIOGRÁFICA}

De forma similar às infecções por SARS-COV ou MERS-COV, o SARS-CoV-2 provoca um estado inflamatório exagerado, chamada de "tempestade de citocinas", sendo que isso parece estar relacionado com a gravidade da doença. As alterações de coagulação surgem em decorrência dessa resposta inflamatória, as quais comumente cursam com a formação de trombos em vários órgãos, com redução do fluxo sanguíneo capilar e desenvolvimento de lesões em locais vitais como os pulmões, coração, cérebro e rins (BENHAMOU D, et al., 2020).

Apesar de a maior parte das mulheres grávidas infectadas pelo SARS-CoV-2 não desenvolverem um quadro grave da doença, cerca de $3 \%$ podem cursar com um quadro clínico mais severo, resultando em maior morbidade materna. A hipercoagulação fisiológica, retardo do fluxo venoso, aumento da capacitância venosa, compressão mecânica das veias pélvicas pelo aumento uterino, dano endotelial pela distensão venosa associada ao comprometimento imunológico está relacionada com pior desfecho na infecção por COVID-19 (MAKATSARIYA AD, et al., 2020). Dado este panorama, é justo considerar a população obstétrica como um grupo vulnerável em relação a essa doença (RONNJE L, et al., 2020).

Sabe-se que a cascata de coagulação é ativada em casos de infecções virais, com o intuito de evitar a disseminação do patógeno, sendo que em caso de infecção pelo SARS-CoV-2, a atividade pró-coagulante é aumentada por via do fator tecidual. Em quadros graves da COVID-19, são observadas lesões na microcirculação pulmonar, e potencialmente em outros órgãos, com acometimento endotelial, que favorece a formação de coágulo microvascular e angiopatia. Já na circulação sistêmica, pode ocorrer a formação de trombose nos grandes vasos, devido a hipercoagulabilidade com hiperfibrogenemia (IBA T, et al., 2020).

Com o intuito de preparar o corpo da mulher para o parto e desenvolvimento fetal, são necessárias certas adaptações. Cabe destacar as modificações hematológicas que ocorrem como a maior produção de fatores coagulativos, principalmente, os fatores VIII, IX e X, e do próprio fibrinogênio, $50 \%$ acima do valor sérico normalmente achado. A queda acentuada da atividade fibrinolítica é também notável, tornando a gravidez um fenômeno hipofibrinolítico. Outra mudança que justifica esse deslocamento é o aumento de anticoagulantes endógenos, como a Antitrombina (AT) e a proteína S. Na gravidez, já se espera uma menor Resistência Vascular Pulmonar (RVP), associado a um maior débito cardíaco, aumentado em mais de $40 \%$ do valor prégravidez, sobretudo, após 8 semanas gestacionais, reforçando ainda mais o ambiente pró-trombótico. Dessa maneira, a gestação é fisiologicamente um evento pró-trombótico e hipofibrinolítico (PILLAY OS, et al., 2016).

O perfil pró-coagulante no SARS-CoV-2 mostra-se, repetidamente, com níveis elevados de D-dímero, estabelecido, até o presente momento, como um marcador de trombose nessa doença, esse que representa um produto de degradação do fibrinogênio e do coágulo de fibrina pela plasmina, aparece em situações clínicas como o Tromboembolismo Pulmonar (TEP) e Trombose Venosa Profunda (TVP) (SATHLER PC, 2020).

Além disso, acerca dos órgãos acometidos por microtrombos pós-COVID-19, analisou-se que apesar do receptor da Enzima Conversora de Angiotensina-2 (ECA2), principal receptor de entrada viral, ocorrer em diversos órgãos (rim, coração, fígado, cérebro, ossos, pele), está presente inclusive no principal órgão acometimento pela doença, o pulmão (VINAYAGAM S e SATTU K, 2020).

Deve-se salientar ainda que a lesão hepática está associada a infecção grave por COVID-19, podendo haver disfunção dos hepatócitos e alteração das enzimas hepáticas, sendo o fígado o segundo órgão mais acometido pelo vírus, atrás somente dos pulmões (RONNJE L, et al., 2020). Verifica-se ainda que um Ddímero $>3 \mathrm{mg} / \mathrm{L}$, repetia-se $50-70 \%$ dos casos não recuperados frente à $1-5 \%$ dos casos de bom prognóstico, associou-se piores prognósticos conforme o aumento da dosagem verificada desse marcador (LEVI M e HUNT JB, 2020). 
Em quadros de coagulopatia, pode haver situações trombóticas ou hemorrágicas, e, nesses casos, há um consumo maior do fator de coagulação, devido a ativação concomitante da cascata de coagulação e fibrinolítica (KOUMOUTSEA EV, et al., 2020). A coagulopatia e o aumento do D-dímero são manifestações características na COVID-19, e estão relacionadas com um pior prognóstico da doença (IBA T, et al., 2020).

No que diz respeito à transmissão do vírus por meio da amamentação, ainda não há evidência de que o patógeno possa estar presente no leite materno de pacientes infectadas. Portanto, a preocupação maior é de que a mãe possa transmitir o vírus por meio de gotículas durante a amamentação e não pelo leite materno em si, sendo a continuação ou interrupção do aleitamento decidido conforme preferência da paciente e da equipe médica (PACHECO-ROMERO J, et al., 2020).

Com o avançar da gravidez, tanto o volume de plasma renal quanto à taxa de filtração glomerular aumenta, comparando com mulheres não grávidas, a taxa sobe para $40-65 \%$ e 50-85\%, respectivamente. Além disso, tanto a resistência vascular aferente quanto à eferente aumentam, podendo facilitar o aparecimento de uma hipertensão glomerular (PILLAY PS, et al., 2016). O SARS-CoV-2, ao ligar-se ao receptor ECA2, gera uma desregulação do sistema renina-angiotensina-aldosterona, levando a três eventos que pioram o quadro da coagulopatia: a ativação do sistema complemento, perdurando à hipercoagulabilidade e a microangiopatia, a hipóxia e a hipotensão, ambas podendo resultar em uma insuficiência renal aguda (VINAYAGAM S e SATTU $\mathrm{K}, 2020)$.

Em um estudo que comparava os desfechos de pacientes grávidas e não grávidas acometidas com a Síndrome Respiratória Aguda Grave (SARS ou SARA), na época do surto por SARS-CoV (2002-2004), notouse que o aparecimento da trombocitopenia era maior em mulheres não grávidas, contrário ao que se pensava. Concluiu-se que apesar da infecção existir, essa não demonstrou impactos significativos comparados à dificuldade da própria gravidez (LAM CM, et al., 2004).

Em comparação aos casos graves, a diferença na pesquisa já era bem mais visível, o grupo de mulheres grávidas com SARS era mais acometido por sepse, Coagulação Intravascular Disseminada (CID), necessitava mais de ventilação mecânica e de maiores dias de internação (10 dias a mais que no segundo grupo), além de maior incidência de Insuficiência Renal Aguda (LAM CM, et al., 2004). De acordo com os dados do Centro de Controle e Prevenção de Doenças dos Estados Unidos (EUA), conclui-se algo semelhante, as gestantes acometidas por SARS-CoV-2 não apresentariam um risco maior de morrer, em relação às não gestantes, mas sim, um potencial maior de serem hospitalizadas, necessitando de UTI e ventilação mecânica (D'SOUZA R, et al., 2020).

Ademais, é possível que a SARS-CoV-2 esteja relacionada com a presença de coagulopatia intraoperativa durante partos cesarianos. A teoria foi sugerida após o relato de uma gestante de 26 anos (G1P0), a qual foi submetida a uma cesárea sem saber os resultados do teste para a infecção de COVID-19. Após o procedimento observou-se atonia uterina e sangramento moderado na região, mesmo após a administração de ocitocina, após isso, ocorreu a administração de $0,2 \mathrm{mg}$ de Metilergometrina por via intravenosa, melhorando seus sintomas. Com os resultados em mãos, perceberam que ela era uma paciente assintomática da doença que apresentava um D-dímero e LDH elevados, com fatores de coagulação, plaquetas, TP e INR normais. Observou-se que, quanto mais anormais estiverem os resultados da paciente, maior a chance de sangramento durante o parto, por isso é necessário que o médico responsável tome cuidado e verifique os resultados dos testes de modo a prevenir hemorragias (KINSEY KE, et al., 2020).

Em relação ao manejo de pacientes diagnosticados com COVID-19, sem alteração do D-dímero, do Tempo de Protrombina e do fibrinogênio, a monitoração deve ser diária, e caso haja alteração, é preconizado a monitorização uma ou duas vezes diariamente. De forma geral, é lícito considerar a administração de Heparina de Baixo Peso Molecular (HBPM), já que está relacionada com um melhor prognóstico quanto a mortalidade, dessa forma, promove maior segurança para o estado hemodinâmico do paciente (mortalidade de $64,2 \% \%$ para o grupo que não utilizou HBPM versus 40\%). O sangramento ativo é considerado uma complicação rara da SARS-CoV-2 (THACHIL J, et al., 2020). Vale ressaltar que não é recomendado usar apenas biomarcadores para guiar a conduta cínica, pois isso pode ser prematuro e potencialmente perigoso (D'SOUZA R, et al., 2020). 
Um estudo realizado na França demonstrou que a administração de HBPM com dose profilática em mulheres infectadas, com risco moderado a severo para tromboembolismo (durante o tempo em que os sintomas estão presentes), reduziu os riscos de hemorragia. Entretanto, não há um consenso para essa administração em gestantes, apenas é aconselhado o uso em pacientes que tenham um risco tromboembólico aumentado (independente de infecção) e/ou após cesárea (BENHAMOU D, et al., 2020). Observou-se, também, que a profilaxia deve ser individualizada considerando os seguintes fatores: (1) severidade da doença, (2) se a paciente está no hospital ou isolada em casa, (3) a ocorrência da doença em relação ao tempo do parto, e (4) o risco pró-trombótico, conferido por comorbidades, trombofilias herdadas ou adquiridas, como na gravidez, no período pós-parto, bem como também, atualmente, a infecção por SARS-CoV-2 (D'SOUZA R, et al., 2020).

Na pandemia do COVID-19, observa-se a possibilidade de a resposta inflamatória ser mais severa em gestantes. Esse é um dado de difícil confirmação, já que a maior parte dos estudos realizados ocorre em não gestantes. Além disso, durante uma gestação normal, há o aumento dos valores de D-dímero e da concentração de fibrinogênio, a contagem de plaquetas pode decair, o tempo de ativação parcial de tromboplastina e de protrombina são menores devido ao aumento importante de fatores de coagulação no plasma (BENHAMOU D, et al., 2020). Entretanto, não há valores de referência estabelecidos para avaliar a severidade das coagulopatias em gestantes (D'SOUZA R, et al., 2020).

Observa-se que, em gestantes, há o risco de hipercoagulopatia, mas não há aumento no risco de sangramento, portanto, não há necessidade de correção farmacológica em pacientes que não estão com sangramento expressivo. No entanto, essa hipercoagulopatia, em decorrência da infecção por COVID-19, aumenta expressivamente a morbidade por elevar o risco tromboembolítico, que já é elevado na gestação e no pós-parto (BENHAMOU D, et al., 2020). Através de relatos de caso, foi possível perceber que a SARSCoV-2 também causa processos patológicos na placenta. Isso porque a placenta, quando infectada, apresenta-se muito anormal: no parênquima há o aumento da fibrina perivilosa ( $>30-40 \%$ ) e extensão da placa corial para a placa decidual, redução do espaço interviloso (30-50\%), segundo MONGULA JE, et al. (2020), e deposição de fibrina o que causa necrose do trofoblasto (MAKATSARIYA AD, et al., 2020).

Kadir RA, et al. (2020) relatam que, as alterações fisiológicas inerentes à gestação elevam um possível risco por complicações relacionadas com essa atual doença. As alterações na resposta imune, por exemplo, tornam a gestante relativamente imunocomprometida. Isso deixa ainda mais complexo estabelecer a relação da COVID-19 com a coagulopatia e, por isso, estabelece que, até o momento, os distúrbios coagulativos não parecerem mais favoráveis para grávidas do que em relação à população geral. No entanto, há certas características hematológicas visíveis que se repetem em pacientes severos por COVID-19 como a redução da Contagem de Plaquetas (CP) para abaixo de $150 \times 103$ células $/ \mathrm{mm} 3$, observada em até $80 \%$ dos pacientes que não sobreviveram e até $70 \%$ dos casos dentre os recuperados, sendo que em gestantes esse valor ainda é considerado normal e também um D-dímero elevado, marcador de alta mortalidade com a COVID-19.

Da mesma forma, para Pacheco-Romero J (2020), ainda não há informações suficientes para classificar as mulheres grávidas como um grupo que não apresentaria maior risco em relação à COVID-19, sobretudo, porque as condutas clínicas da doença em si contam com evidências de baixa qualidade, sendo de grau $D$ ou nível 3 e 4 . Dessa maneira, apesar das noções fisiológicas do estado hipercoagulativo na gravidez ser bem estabelecido, ainda são necessários estudos para que seja possível estabelecer a relação do SARSCoV-2 com a gestação.

\section{CONSIDERAÇÕES FINAIS}

As alterações imunológicas, hematológicas e cardiovasculares que ocorrem naturalmente na gestante podem predispor ao agravamento da infecção pelo SARS-CoV-2, principalmente em decorrência de modificações coagulativas. Dessa forma, a gestação é uma condição em que há um estado hipercoagulativo e hipofibrinolítico, e isso, somado a uma condição hiperinflamatória característica da COVID-19 aumenta a chance de morbimortalidade materna. No entanto, notou-se uma carência de estudos e protocolos que informem claramente a relação entre gestação e infecção pelo novo coronavírus, sendo que muitos dados 
são conflitantes sobre o estabelecimento do manejo adequado para pacientes obstétricas infectadas pelo vírus. Portanto, apesar da carência de dados, tem-se claramente estabelecido que se deve haver uma maior atenção para esta população, uma vez que as alterações fisiológicas da gestação podem aumentar o risco de agravamento da COVID-19, principalmente em grávidas que apresentam morbidades.

\section{REFERÊNCIAS}

1. BENHAMOU D, et al. Coagulation changes and thromboembolic risk in COVID-19 obstetric patients. The Obstetric Anaesthesia and Critical Care Club (Club Anesthesie Reanimation en Obstetrique [CARO]), 2020; 39: 351-353.

2. BENHAMOU D, et al. Coagulation changes and thromboembolic risk in COVID-19 pregnant patients, Anaesthesia Critical Care and Pain Medicine, 2020; 39 (3) 351-353.

3. D'SOUZA R, et al. A critical review of the pathophysiology of thrombotic complications and clinical practice recommendations for thromboprophylaxis in pregnant patients with COVID-19, 2020; 99: 1110:1120.

4. IBA T, et.al. Coagulopathy of Coronavirus Disease. Crit Care Med,2020; 48: 1358-1364.

5. KADIR R.A, et al. COVID-19 coagulopathy in pregnancy: Critical review, preliminary recommendations, and ISTH registry - Communication from the ISTH SSC for Women's Health. J Thromb Haemost, 2020; 18: 3086-3098.

6. KINSEY KE, et al. Intraoperative coagulopathy during cesarean section as an unsuspected initial, 2020. BMC Pregnancy and Childbirth, 2020; 20:481.

7. KOUMOUTSEA EV, et al. COVID-19 and acute coagulopathy in pregnancy, Canada. J Thromb Haemost, 2020; 18: 1648-1652.

8. LAM CM, et al. A case-controlled study comparing clinical course and outcomes of pregnant and non-pregnant women with severe acute respiratory syndrome, $2004 ; 111: 771-774$.

9. LEVI M, HUNT BJ. Thrombosis and coagulopathy in COVID-19: An illustrated review. Research and Practice in Thrombosis and Haemostasis, 2020; 4 (5): 744-751.

10. MAKATSARIYA AD, et al. Thrombotic microangiopathy, DIC-syndrome and COVID-19: link with pregnancy prothrombotic state, The Journal of Maternal-Fetal \& Neonatal Medicine, 2020; 6: 1-9.

11. MONGULA JE, et al. COVID-19 during pregnancy: non-reassuring fetal heart rate, placental pathology and coagulopathy, 2020. 56: 773-766.

12. PACHECO-ROMERO J. La incógnita del nuevo coronavirus, la gestante y su niño Lo que el ginecobstetra está conociendo. Rev Peru Ginecol Obstet, 2020; 66 (2): 00005.

13. PILLAY PS, et al. Physiological changes in Pregnancy, Africa. Cardiovascular Journal of Africa, 2016; $27: 89-94$.

14. RONNJE L, et al. Complicated COVID-19 in pregnancy: a case report with severe liver and coagulation dysfunction promptly improved by deliver. BMC Pregnancy Childbirth, 2020; 20: 511.

15. SATHLER PC. Alterações hemostáticas no COVID-19: Uma revisão guiada. Anais da Academia Brasileira de Ciências, 2020. 92 (4): 20200834.

16. VINAYAGAM S, SATTU K. SARS-CoV-2 and coagulation disorders in different organs. Life Sciences, 2020; 260: 118431.

17. THACHIL J, et. al. ISTH interim guidance on recognition and management of coagulopathy in COVID-19. J Thromb Haemost, 2020; 18: 1023-1026.

18. WORLD HEALTH ORGANIZATION (WHO). Coronavirus Disease (COVID 19) Pandemic, 2020 Disponível em: https://www.who.int/emergencies/diseases/novel-coronavirus-2019. Acessado em: 18 de janeiro de 2021. 